\title{
Téoros
}

Revue de recherche en tourisme

\section{La gestion d'un parc à thèmes et la préoccupation pour la clientèle}

\section{Thierry Coltier}

Volume 12, numéro 3, octobre 1993

Le renouveau des parcs à thèmes

URI : https://id.erudit.org/iderudit/1077937ar

DOI : https://doi.org/10.7202/1077937ar

Aller au sommaire du numéro

Éditeur(s)

Université du Québec à Montréal

ISSN

0712-8657 (imprimé)

1923-2705 (numérique)

Découvrir la revue

Citer cet article

Coltier, T. (1993). La gestion d'un parc à thèmes et la préoccupation pour la clientèle. Téoros, 12(3), 37-38. https://doi.org/10.7202/1077937ar d'utilisation que vous pouvez consulter en ligne.

https://apropos.erudit.org/fr/usagers/politique-dutilisation/ 


\section{La gestion d'un parc à thèmes et la préoccupation clientèle}

\section{Thierry Coltier*}

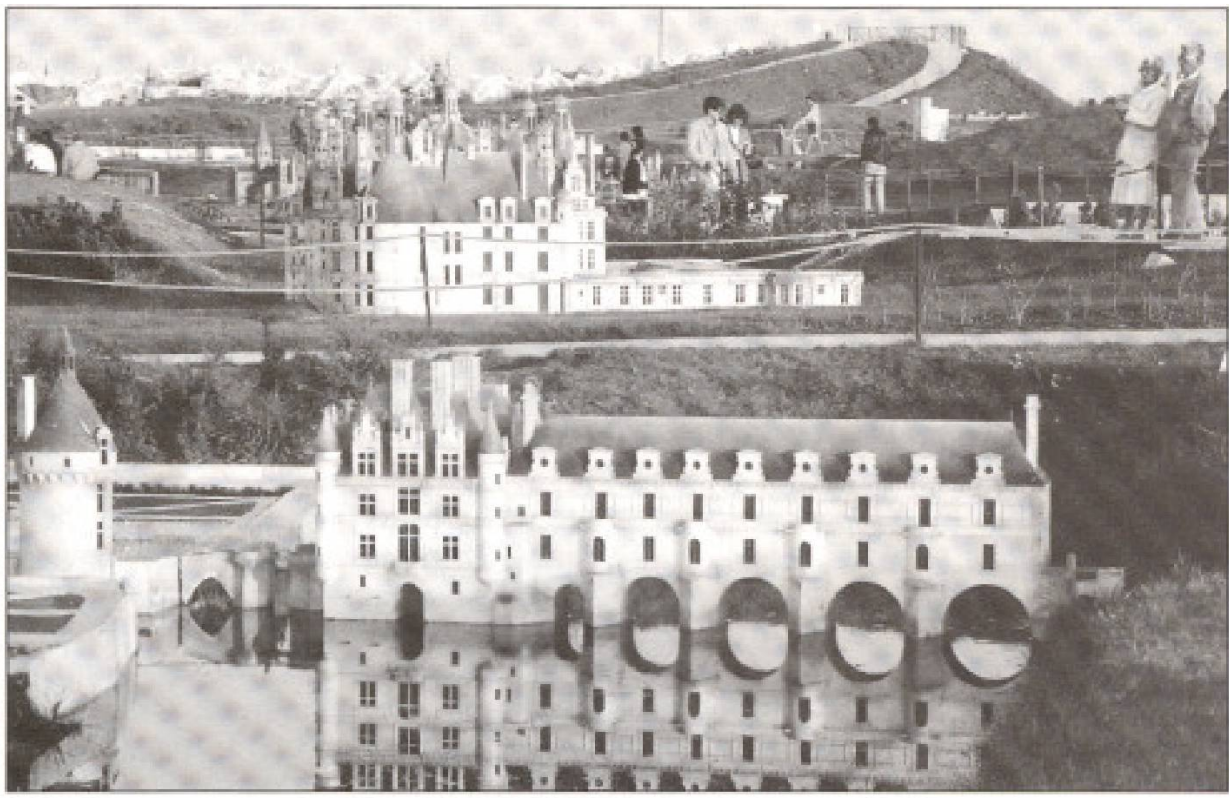

La population françaisen'estpas encore familiarisée avec le concept du *parcà thèmess. L'avènement récent d'EuroDisney, en attirant les projecteurs de l'actualité, a donné un éclairage nouveau à cette forme de loisirs. Mais, si une partie de mes concitoyens commence à faire la différence entre des parcs dont l'importance et les thèmes sont aussi éloignếs qu'EuroDisney, le Futuroscope ou France Miniature, pour la majorité d'entre eux, le parc à thèmes n"est guère qu'une sorte de fête foraine sédentaire, améliorée parce que plus propre et mieux fréquentée.

Aussi, la première des préoccupations du gestionnaire d'un parc est d'affirmer la particularité du concept dont il a la charge. Ce qui n'est pas toujours facile, en particulier lorsque le thème est vague et qu'il est composé de sous-thèmes hybrides, sans réelle cohésion avec le thème principal.

Il lui faut ensuite *positionners son parc dans un gamme d'équipement de loisirs allant en gros du parc d'attractions foraines au musée de sciences et technologies ou à l'écomusée. Ce positionnement, qui doit être le reflet le plus objectif du produit réalisé, a des conséquences sur la politique tarifaire et le ton de la communication.

En dépit de tout cela, le visiteur, lorsqu'il arrive, n'a qu'une idée assez vague de ce qu'il va voir. Tant mieux! Il fauten profiter pour le surprendre dès l'entrée. De l'autre côté du miroir, tout n'est qu'ordre et beauté... On le sait, les parcs à thèmes veulent étonner et dépayser, amuser et charmer, instruire et séduire... tout le monde! On est venu en famille et chacun, quelque soit son âge et quelque soit son niveaude culture, doity trouver soncompte.

A y regarder de près, rares sont les occasions qui permettent à la famille entière de communier dans une même pratique de loisirs. Etre bien ensemble est souvent une des premières motivations de la visite familiale d'un parc à thèmes.

Le concepteur doity penser au moment de la création en réalisant un cadre aussi enchanteur que convivial (accès handica- 
pés, point change bébé, menus de restaurant traduits en langues étrangères, etc.).

S'il aime être dépaysé, le visiteur a néanmoins besoin de trouver ses repères. Les services habituels font partie de l'accueil, si important dans une activité touristique. Maintenir dans la durée une bonne qualité d'aocueil, nécessite, pour le personnel en contact avec le public, une formation initiale. Par la suite, il faut tout au long de la saison, entretenir le sentiment que l'accueil est important. Un des moyens est de procéder à des enquêtes périodiques auprès des visiteurs.

Leur demander leur avis sur l'accueil des caissières et des hôtesses, la qualité des espaces verts ou de la restauration permet, si l'information remonte bien, que chaque service dispose d'un baromètre et qu'il prenne conscience que la satisfaction du visiteur résulte d'un tout qui implique chacun.

En région parisienne, l'ouverture d'EuroDisney a rehaussé les standards de qualité del'accucil. En effet, ce parcn'a pas son pareil pour donner au visiteur, tous les jours de l'année, l'impression qu'il est venu un jour de fête.

Les parcs récréatifs, mais aussi les musées, accordent de nouveaux efforts à l'accueil. Ils ont raison car c'est une composante essentielle de la satisfaction du visiteur et il ne faut jamais oublier que le bouche-àoreille est le premier vecteur de la communication d'un équipement touristique.

Fairevenir la dientèleest bien sûr la préoccupation fondamentale del'exploitant. Les parcs à thèmes mobilisent des financements considérables pour leur réalisation, les investisseurs attendent une rentabilité des fonds investis, spécialement lorsque la réalisation est financée par des fonds privés. On soulignera au passage *la concurrence déloyales qui existe de fait dans le monde des musées, comme celui des parcs à thèmes, lorsqu'un équipement, financé par des fonds publics, n'a pas à se soucier de rentabiliser l'investissement et bénéficie parfois de subventions d'exploitation... Tous ces équipements s'adressent à un même marché, sen sible au rapport qualitéprix. Les parcs privés sont souvent obligés de compenser, par un dynamisme commercial, lui aussi coûteux, la perte de visiteurs sollicités par les équipements publics dispensés de pratiquer la vérité des prix.
Néanmoins, cette remarque est valable à propos du marché touristique ou le marché des groupes n'effectuant qu'une à deux sorties par an, car pour ce qui est du marché de la population résidentielle, il est vrai que la synergie entre les équipements joue. Un visiteur ayant apprécié sa visite sera enclin à programmer la visite d'un site comparable. Dans le contexte du marché enoore naissant des parcs à thèmes, les exploitants ont tout à gagner en encourageant leurs visiteurs à se rendre sur des sites concurrents. Nombreux sont ceux qui l'ont compris et diffusent, sous forme de réciprocité, la documentation de leurs confrères. Ils se regroupent même parfois en associations, afin de promouvoir ensemble leur image et proposer à leurs visiteurs des avantages tarifaires chez les autres.

Visiter un parcà thèmesn'est pas un acte de consommation ordinaire. C'est une démarche qui répond à une envie occasionnelle résultant de facteurs divers. Facteurs parmi lesquels la météo occupe une place prépondérante. Il s'ensuit souvent une affluence qui se retrouve à certaines heures de certains jours. S'organiser pour permettrenéanmoins une visite agréable, avec un accueil de qualité, est également un devoir de l'exploitant. Force est de constater que ni l'information ni les incitations tarifaires, ne permettent wraiment d'étaler dans le temps la fréquentation qui demeure, pour certains parcs, un problème important, prejudiciable à leur image.

Faire venir et revenir. Inciter les gens à revenir, deux moyens sont généralement mis en oeuvre: réaliser d'une part des événements durant la saison, et renouveler d'autre part, une partie du produit d'une année sur l'autre. L'exploitation du questionnaire des visiteurs doit orienter la politique de réinvestissement, en s'efforçant de satisfaire les demandes particulières de certaines catégories de visiteurs.

L'évolution observée ces dernières années à propos des nouveaux parcs ou de nouvellesattractions, montrequ'il ya une tendance au rapprochement entre les parcs à thèmes et les musées.

Les premiers développent des concepts dont le caractère culturel et pédagogique s'affermit. Les seconds s'efforcent de présenter leurs collections de manière de plus en plusinteractive et ludique. Il y a tout lieu de penser qu'il s'agit là d'un phénomène qui s'accentuera dans les années à venir et que la frontière entre les uns et les autres sera de moins en moins rigide. La culture accessible à tous réconciliera le monde, celui de la «culture avec celuides \& loisirs pour la plus grande satisfaction de chacun de nous. $f$

\section{Notes}

Le monde dee parcs: per apectives etrólites, dossier réalisé en collaboration avec le Groupe Eiffel, décembre 1985, $100 \mathrm{p}$.

Annuaire dea intervenants en matibre de pares de loisirs - Bureaux d'otudes, architectes. gestionnaires, fournieseure, Investisesurs.... pour le compte du ministere du Tourisme.

Epot: un phalanstave du futur?, publication du CCl (Centre Georges Pompidou], in Payage: parce urbaine et uburbaine, mars 1988

Les parcs rechatifs, serie de trois articles pour la revue Espaces.

Les parcs recreatifs, un produit nouvesu, article pour L'Encyclopaedia Univerealis, mai 1989. 